\title{
Conventional and quantitative EEG in the diagnosis of delirium among the elderly
}

\author{
Sandra A Jacobson, Andrew F Leuchter, Donald O Walter
}

\begin{abstract}
This study was performed to determine whether an admission quantitative EEG (QEEG) could assist in the differential diagnosis of encephalopathy among a group of elderly subjects with delirium, dementia, and delirium coexistent with dementia. Thirty four subjects from 57 to 93 years had standard 17-channel EEG and quantitative EEG studies, using a linked-ear reference. EEGs were independently rated by two electroencephalographers blind to clinical diagnosis, using conventional criteria to assess the degree of encephalopathy. Brain maps were scored by a scale developed by the authors. Numerical data examined included mean posterior dominant frequency, absolute and relative power in the delta, theta and alpha bands, and slowwave ratios. The grouping of experimental subjects was by the discharge diagnosis, made using DSM-III-R criteria. Stepwise discriminant analysis was performed to determine which EEG and QEEG variables were best able to distinguish cases. Variables which collectively distinguished normal from encephalopathic records were Mini-Mental State Examination scores and relative power in the alpha frequency band. Variables which collectively distinguished delirium from dementia were EEG theta activity, relative power in delta, and brain map rating. The results suggest that cross-sectional QEEG study is potentially useful in the early differential diagnosis of encephalopathy, and that the variables which distinguish normal from encephalopathic patients might differ from the variables which distinguish delirium from dementia.
\end{abstract}

(₹ Neurol Neurosurg Psychiatry 1993;56:153-158)

The syndrome of delirium has long been understood to be symptomatic of serious underlying illness in the elderly, and it is well established that early diagnosis of this syndrome is critical. ${ }^{1}$ A growing literature demonstrates the significance of delirium as a problem among elderly patients admitted to hospital in terms of increased morbidity, mortality, and length of hospital stay in delirious compared with non-delirious patients. ${ }^{1-3}$ Considering its clinical importance, it is a problem that delirium often goes undiagnosed, misdiagnosed, or mistaken for other disorders, such as an exacerbation of dementia or depression. ${ }^{4}$

Unlike most other psychiatric disorders, for delirium there are several laboratory tools available to assist in differential diagnosis and monitoring of therapy, but these tools, particularly the EEG, are currently underused for this purpose. The standard in current use of the diagnosis of delirium is the clinical examination, used in conjunction with DSM-III-R criteria. ${ }^{5}$ In addition, the Mini-Mental State Examination (MMSE) developed by Folstein et al is commonly used, ${ }^{6}$ and at present is the best and most easily administered of the tests available. As Macdonald et $a^{7}$ have noted, the validity of serial use of this instrument has not been tested.

Assessment of delirium among the elderly would best be accomplished by a measure which would not only distinguish "organic" from "functional" causes of acute mental status changes, but also differentiate delirium from dementia. Ideally, the measure would also provide a reliable indicator of severity of illness, and retain its validity with serial use. EEG with quantitative analysis has the potential to fulfil these requirements.

The utility of the conventional EEG in the diagnosis and monitoring of delirium has been well established since the 1940s, when Romano and Engel ${ }^{8}$ studied the relationship between level of arousal and degree of electroencephalographic abnormality among delirious patients admitted to hospital. They found that decreased background EEG frequency and disorganisation of the EEG were correlates of reduced arousal. Subsequent work by Laidlaw and Read, ${ }^{9}$ Obrecht $e t a l,{ }^{10}$ and Jerrett and Corsak $^{11}$ replicated those findings, and extended this work by the use of the computeranalysed EEG (also known as quantitative EEG or QEEG).

Trzepacz et al ${ }^{12}$ examined a cohort of liver transplantation candidates using a test battery which included EEG, MMSE, and Trails A and $B$ testing. On the basis of these measures, they developed a discriminant function which enabled them to identify delirious patients in their sample with greater than $95 \%$ accuracy.

Koponen et $a l^{13}$ compared QEEG findings from elderly delirious patients with those of healthy, community-dwelling elderly control subjects on variables of relative power, power ratios, occipital peak frequency, and mean frequency. They found significant differences in the EEG spectra of the two groups; specifically, they noted that reductions in the proportion of alpha activity and in mean frequency 
were associated with declining cognitive function as measured by MMSE, and that increases in the proportion of delta activity were associated with increased length of delirium and hospital stay. They also observed that delirious subjects with coexisting dementia consistently showed the most abnormal EEGs. This work has shown that there are consistent and recognisable electrophysiological abnormalities in delirium which are readily detected by EEG, particularly when supplemented by quantitative analysis.

Before these tools can be recommended for use outside the research electrophysiology laboratory, a few questions must be answered. First, can the EEG and/or QEEG reliably distinguish "organic" from "functional" causes of acute changes in mental status? Do they add anything to the clinical examination in the diagnosis of delirium? The Trzepacz study ${ }^{12}$ does address this question, but only for one particular group of patients, and using conventional EEG only. Second, can the QEEG reliably distinguish delirium from other forms of encephalopathy, such as dementia? None of the reported studies looked at this question; the Koponen group, although closest to it, did not separate dementia (without delirium) as a group for comparison. It seems obvious that on serial study, delirium and dementia could be distinguished, but whether the EEG is sufficiently different on cross-sectional analysis to be distinguishable is of even greater clinical interest. We performed the current pilot study to examine these questions.

\section{Methods}

Subjects

Four groups of subjects were studied: inpatients with delirium, inpatients with delirium coexisting with dementia (referred to as the "combined disease" group), outpatients with dementia, and outpatient controls. Inpatient subjects were identified from records maintained by the Clinical Electrophysiology Laboratory for the period 1988 to 1991 . Records on all patients with referring diagnoses of encephalopathy (including delirium, dementia, or organic mental syndrome) who had undergone conventional as well as QEEG studies were selected. Seventy nine such records were identified, and charts for these patients were reviewed to determine discharge diagnoses, which had been made using DSM-III-R criteria. Most patients were not considered further because of uncertain diagnosis, or confounding factors such as intervening electroconvulsive shock therapy or surgery. Eight subjects with delirium as the sole diagnosis were identified, and were assigned to the "delirium" group. Ten patients with delirium coexisting with dementia (also diagnosed using DSM-III-R criteria) in the absence of other major psychiatric diagnoses were identified, and were assigned to the "combined disease" group. None of the inpatients studied had a sole discharge diagnosis of dementia.

Nine subjects with dementia and seven community-dwelling control subjects were drawn from an ongoing dementia study at the same site. ${ }^{14}$ Subjects were excluded from that study for any history of alcohol abuse, head trauma, concurrent or past central nervous system disease other than dementia, serious physical illness, or other psychiatric illness.

Demographic and diagnostic information for each study subject are displayed in table 1 . Patients ranged in age from 57 to 93 , and control subjects from 64 to 79 . The average age in the delirium group was 74 years, in the dementia group 78 years, and that of control subjects 71 years. Fourteen of eighteen subjects in the delirium and combined disease groups and none of those in the dementia group were receiving medications for symptom control. Control subjects were not all free of medical problems such as hypertension or hyperthyroidism.

\section{Procedures}

All subjects had a standard clinical EEG. ${ }^{14}$ Data were recorded from 17 channels, with active electrode placement at FP1, FP2, F3, F4, F7, F8, C3, C4, T3, T4, T5, T6, P3, P4, $\mathrm{O} 1$, and $\mathrm{O} 2$, using a linked-ear reference. The seventeenth channel was used for eye movement monitoring. QEEG data were collected while subjects were resting with eyes closed, and were digitalised and analysed by a QSI 9000 system. Frequency bands were defined as follows: delta $0-4.0 \mathrm{~Hz}$, theta 4.0 to $8.0 \mathrm{~Hz}$, and alpha 8.0 to $12.0 \mathrm{~Hz}$. The specific protocol for collection of data, including standards for maintaining subject alertness, have been described elsewhere. ${ }^{14}$ For this study, four map displays were created for each QEEG session, with two different parameters and scale maxima: absolute power with maxima of 103 microvolts-squared and 215 microvoltssquared, and relative power (that is, absolute power in a band as a fraction of total power) with maxima of $75 \%$ and $100 \%$. On the day of each EEG/QEEG study, mental status was assessed by the Folstein Mini-Mental State Examination. For six of the subjects (flagged in table 1), MMSE was not performed on the day of the EEG study, but was estimated from clinical notes on cognitive status in the medical record for those dates.

Independently and without knowledge of the subject's clinical diagnosis, two electroencephalographers rated each EEG study, using conventional EEG criteria to assess the degree of encephalopathy, including estimated posterior dominant frequency and amount of slow wave activity in the theta and delta frequency ranges (scored as $0=$ none, $1=$ minimal amount, 2 = moderate amount, 3 = large amount). In addition, the Mayo Clinic classification criteria ${ }^{15}$ were used to assign an overall dysrhythmia score to each EEG study.

The two electroencephalographers also rated the brain maps, using a scale developed by the authors. This scale is based on the criterion currently used in our laboratory to assess the degree of encephalopathy in clinical mapping studies; namely, the amount of activity in the slow wave bands relative to the alpha band. The scale used was as follows: excess generalised delta-none $=0$; mild (delta < 
Table 1 Individual case data

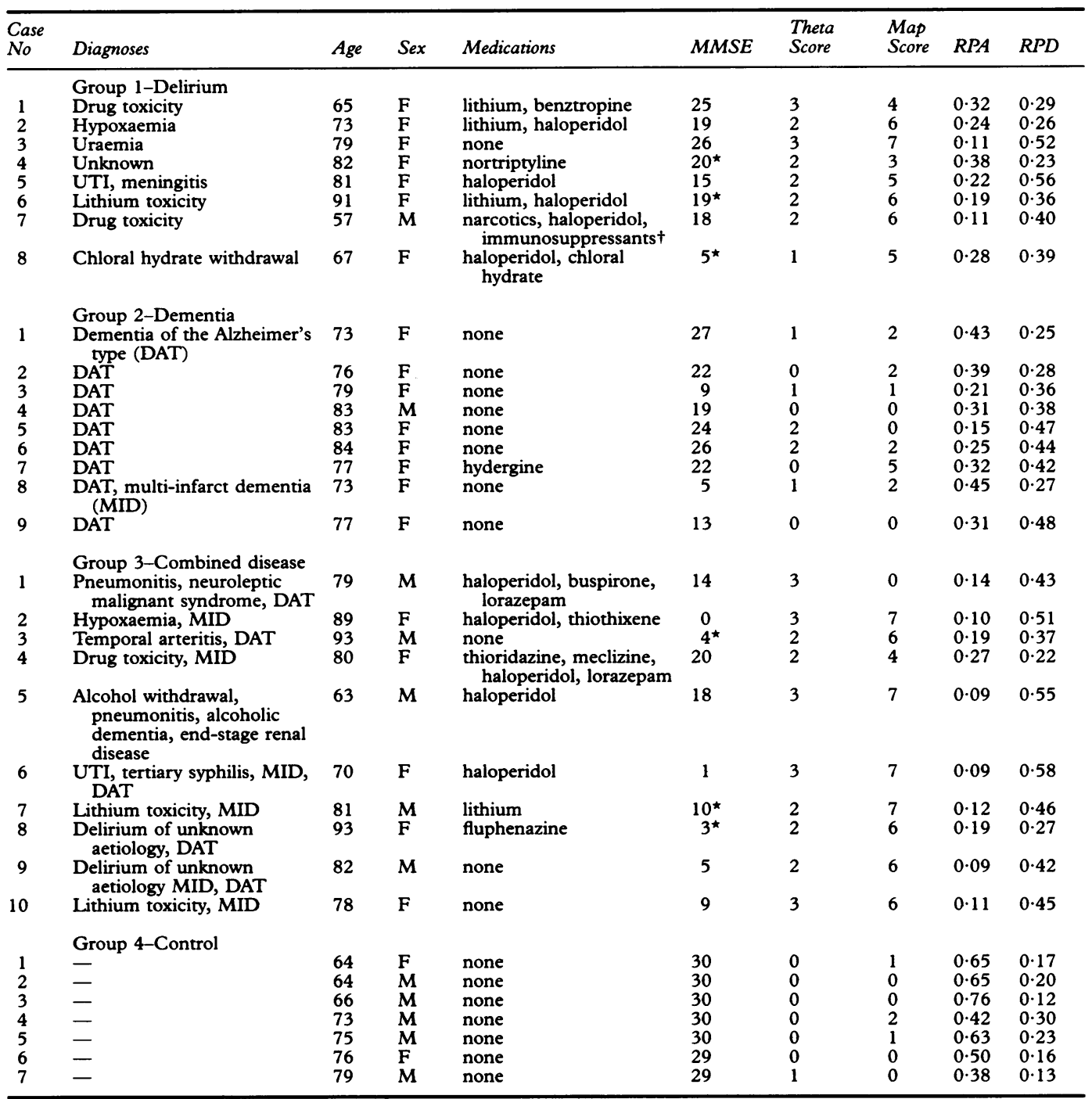

* MMSE estimated from information on cognitive status noted in medical record.

+ Immunosuppressants included: cyclosporine, azathioprine, leucovorin, nystatin and prednisone.

alpha $)=1 ;$ moderate $($ delta $=$ alpha $)=2$; severe (delta $>$ alpha) $=3$; extreme (delta $>$ alpha and delta $>$ theta) $=4$. Excess generalised theta - none $=0$; mild (theta $<$ alpha) $=$ 1 ; moderate (theta $=$ alpha) $=2$; severe (theta $>$ alpha) $=3$. The scores for excess delta and theta were added for a total brain map slowwave excess score, with a maximum score of 7 . Abnormalities in the form of focalities or asymmetries were ignored. Very high interrater reliability was attained in our laboratory, with all map scores agreeing within one scale point.

In addition to maps, numerical data were extracted from each QEEG study. The following variables were examined: mean posterior dominant frequency (defined as the average of the mean frequencies in the alpha band obtained for the electrode positions T5, T6, $\mathrm{P} 3, \mathrm{P} 4, \mathrm{O} 1, \mathrm{O} 2$ ), total absolute power in delta, theta and alpha (defined as the average of mean absolute power in each frequency band, obtained for all electrode positions, excluding F1 and F2), relative power in delta, theta and alpha (defined as the total absolute power in each frequency band divided by the summed absolute power of all three bands), and slowwave ratios (defined as the total absolute power in the alpha frequency band divided by total summed absolute power in the delta plus theta bands).

\section{Statistical analysis}

To determine which EEG and QEEG variables were most useful in distinguishing "normal" from "abnormal" records, and "delirium" from "dementia" (in the absence of delirium), two stepwise discriminant analyses were performed using BMDP $7 \mathrm{M} .{ }^{16}$ This programme determines the combination of variables which best predicts the group to which a case belongs, evaluates the number of cases correctly classified, and performs a jackknife validation procedure to reduce the bias in this evaluation. The "jackknife" is a subtype of the cross-validation method. ${ }^{16}$ In the first analysis, the delirium, dementia, and combined-disease groups were combined to determine which group of variables most accurately identified cases as "normal" or "abnormal".

In the second analysis, the delirium and the combined-disease groups were combined, and 
the control group was omitted, to determine which group of variables most accurately identified cases as "delirium" or "dementia". It should be noted that, in this latter categorisation, some of the cases in the "delirium" group also had dementia; the purpose here was to identify delirium, whether or not it coexisted with dementia.

\section{Results}

Table 2 displays group mean and standard deviation values for the variables identified as most useful in record categorisation. For the variables MMSE and relative power in alpha (RPA), the higher the score, the more normal the study; for theta score, map score (total slow-wave excess), and relative power in delta (RPD), the lower the score, the more normal. As can be seen from this table, the combined disease group (delirium coexisting with dementia) showed the most abnormal values.

In the first analysis, variables identified as most useful in distinguishing "normal" from "abnormal" (or "encephalopathic") cases included MMSE and relative power in the alpha frequency band. MMSE alone identified $85 \%$ of all cases correctly, but misidentified $19 \%$ of encephalopathic cases as normal. The combination of MMSE and relative power in alpha achieved a $94 \%$ correct identification overall, with only $4 \%$ of encephalopathic records misidentified as normal. (Wilks' Lambda 0.36, Approximate F-statistic 26.92, DF2, 31). Table 3 shows the results of the jack knifed classification for this analysis.

In the second analysis, variables identified as most useful in distinguishing cases of "delirium" from "dementia" (in the absence of delirium) included amount of EEG theta activity, relative power in the delta frequency band, and the brain map rating (absolute power with scale maximum of 103 microvoltssquared). This combination of variables achieved a $93 \%$ correct identification of cases, with $11 \%$ of delirium cases misidentified as dementia. (Wilks' Lambda 0.25, Approximate F-statistic $22 \cdot 73, \mathrm{DF} 3,23$ ) Table 4 shows the results of the jack knifed classification for these variables considered separately as well as in combination. Note that theta alone was able to classify nearly $89 \%$ of cases correctly, with only $6 \%$ of delirium cases misclassified as dementia.

\section{Discussion}

The results of this pilot study suggest that EEG with quantitative analysis has considerable potential use in a diverse group of patients with encephalopathy, not only in confirming the clinical diagnosis of an organic syndrome, but in distinguishing delirium from dementia. In addition, the analyses indicate that the specific EEG variables which distinguish encephalopathy from normal or "functional" states might differ from the specific variables distinguishing delirium from dementia.

The variables which emerged from this exploratory study best able to distinguish normal from encephalopathic cases included MMSE and relative power in the alpha frequency band. It would be difficult to draw any inference regarding MMSE as a predictor from this study, since the controls were healthy community-dwelling subjects who had uniformly high scores. Although, in principle, the same argument could be applied to the variable of relative power in alpha, what was actually observed was that there was a good range of values around the mean for this variable in the control group. For future studies, it will be necessary to identify a more appropriate control group than extremely healthy elderly outpatients, but it is not obvious how an appropriate group could be constituted, since many of the illnesses which necessitate admission to hospital are associated with their own abnormal EEG findings.

The variables which were best able to distinguish delirious from non-delirious patients included the amount of EEG theta activity, relative power in the delta frequency band, and the brain map rating. These variables could be useful in determining whether delirium is present, given that the clinical examination and EEG have been found abnormal on preliminary analysis. This determination has definite clinical use as the presence of delirium necessitates an immediate, costly, and at times, invasive work-up for precipitating causes.

The brain map which showed the best predictive capability was the map of absolute power with the scale maximum of 103 microvolts-squared, which is the more sensitive setting. The emergence of the brain map rating as a distinguishing variable indicates that it might be worthwhile to test further the map scoring system developed for this study. This

Table 2 Group mean (SD) values. Selected variables *

\begin{tabular}{|c|c|c|c|c|c|c|c|c|c|c|}
\hline & \multicolumn{2}{|c|}{$M M S E$} & \multicolumn{2}{|l|}{ Theta } & \multicolumn{2}{|c|}{ Map score } & \multicolumn{2}{|l|}{$R P A$} & \multicolumn{2}{|l|}{$R P D$} \\
\hline & Mean & $(S D)$ & Mean & $(S D)$ & Mean & $(S D)$ & Mean & $(S D)$ & Mean & $(S D)$ \\
\hline $\begin{array}{l}\text { Delirium group (1) } \\
\text { Dementia group (2) } \\
\text { Combined disease group (3) } \\
\text { Groups } 1+3 \\
\text { Control group (4) }\end{array}$ & $\begin{array}{r}18 \cdot 3 \\
18 \cdot 5 \\
8 \cdot 4 \\
12 \cdot 8 \\
29 \cdot 7\end{array}$ & $\begin{array}{l}6 \cdot 5 \\
7 \cdot 7 \\
7 \cdot 0 \\
8 \cdot 3 \\
0 \cdot 4\end{array}$ & $\begin{array}{l}2 \cdot 1 \\
0 \cdot 7 \\
2 \cdot 5 \\
2 \cdot 3 \\
0 \cdot 1\end{array}$ & $\begin{array}{l}0 \cdot 6 \\
0 \cdot 8 \\
0 \cdot 5 \\
0 \cdot 5 \\
0 \cdot 3\end{array}$ & $\begin{array}{l}5 \cdot 2 \\
1 \cdot 5 \\
5 \cdot 6 \\
5 \cdot 4 \\
0 \cdot 5\end{array}$ & $\begin{array}{l}1 \cdot 2 \\
1.5 \\
2 \cdot 1 \\
1 \cdot 7 \\
0 \cdot 7\end{array}$ & $\begin{array}{l}0 \cdot 23 \\
0 \cdot 31 \\
0 \cdot 14 \\
0 \cdot 18 \\
0 \cdot 57\end{array}$ & $\begin{array}{l}0 \cdot 09 \\
0 \cdot 09 \\
0 \cdot 06 \\
0 \cdot 09 \\
0 \cdot 13\end{array}$ & $\begin{array}{l}0 \cdot 38 \\
0 \cdot 37 \\
0 \cdot 43 \\
0 \cdot 40 \\
0 \cdot 19\end{array}$ & $\begin{array}{l}0 \cdot 11 \\
0 \cdot 08 \\
0 \cdot 11 \\
0 \cdot 11 \\
0 \cdot 06\end{array}$ \\
\hline
\end{tabular}

* MMSE is the Mini-Mental State Examination score.

Theta refers to amount of activity in the $4-8 \mathrm{~Hz}$ range on conventional EEG, scored from 0 to 3 , as described in text Map score is a rating of total slow-wave excess on brain map display.

RPA is relative power in the alpha band, from QEEG.

RPA is relative power in the alpha band, from QEEG. 
Table 3 Normal vs encephalopathic records (groups 1,2, and 3 versus 4) jackknifed classifications

\begin{tabular}{|c|c|c|c|c|c|}
\hline & \multicolumn{2}{|c|}{$\begin{array}{l}\text { Encephalopathic records } \\
\text { Number classified as: } \\
\text { Enceph }\end{array}$} & \multicolumn{2}{|c|}{$\begin{array}{l}\text { Normal records } \\
\text { Number classified as: }\end{array}$} & $\begin{array}{l}\text { Total } \\
\% \\
\text { correct }\end{array}$ \\
\hline $\begin{array}{l}M_{M S E^{\star}} \text { alone } \\
\text { RPA }^{\star} \text { alone } \\
\text { MMSE + RPA }\end{array}$ & $\begin{array}{l}22 \\
25 \\
26\end{array}$ & $\begin{array}{l}5 \\
2 \\
1\end{array}$ & $\begin{array}{l}0 \\
1 \\
1\end{array}$ & $\begin{array}{l}7 \\
6 \\
6\end{array}$ & $\begin{array}{l}85 \cdot 3 \\
91 \cdot 2 \\
94 \cdot 1\end{array}$ \\
\hline
\end{tabular}

$\star \mathrm{Nl}=$ normal.

MMSE = Mini-Mental State Examination.

$\mathrm{RPA}=$ relative power in the alpha band, from QEEG.

Table 4 Delirious vs non-delirious records (groups 1 and 3 versus 2) jackknifed classifications

\begin{tabular}{|c|c|c|c|c|c|}
\hline & \multicolumn{2}{|c|}{$\begin{array}{l}\text { Delirious records } \\
\text { Number classified as: }\end{array}$} & \multicolumn{2}{|c|}{$\begin{array}{l}\text { Non-delirious records } \\
\text { Number classified as: }\end{array}$} & $\begin{array}{l}\text { Total } \\
\% \\
\text { correct }\end{array}$ \\
\hline & & & & & \\
\hline Theta ${ }^{\star}$ alone & 17 & $\frac{1}{2}$ & 2 & $\begin{array}{l}7 \\
8\end{array}$ & $\begin{array}{l}88 \cdot 9 \\
88.9\end{array}$ \\
\hline $\begin{array}{l}\text { Map score ^ alone } \\
\text { RPD }^{\star} \text { alone }\end{array}$ & 11 & 7 & 4 & $\begin{array}{l}0 \\
5\end{array}$ & $59 \cdot 3$ \\
\hline Theta + Map & 16 & 2 & 1 & 8 & 88.9 \\
\hline Map + RPD & 16 & 2 & 1 & 8 & 88.9 \\
\hline Theta + Map + RPD & 16 & 2 & 0 & 9 & $92 \cdot 6$ \\
\hline
\end{tabular}

$\star$ ND $=$ Non-delirious.

Theta refers to amount of activity in the $4-8 \mathrm{~Hz}$ range on conventional EEG, scored from 0 to 3 , as described in text.

Map score is a rating of total slow-wave excess on brain map display.

RPD is relative power in the delta band, from QEEG

system takes into account slow-wave power relative to alpha power, so represents another way to express relative power. This is likely to be a critical point, as individual subjects apparently differ at baseline in terms of how much total power is generated. In encephalopathy, a subject with a moderate excess of slow-wave power accompanied by a moderate amount of alpha power might prove to be less impaired than a subject with a mild excess of slow-wave power accompanied by little or no alpha power. In any event, more experience with this map rating system is needed. A standardised system of map interpretation would be of obvious value, particularly if brain mapping were to become more widely used outside research EEG settings.

Generally, these study findings agree with those of Koponen $e a^{13}$ in that relative power was found to be an important variable distinguishing encephalopathic from normal cases, and that the most abnormal EEG and quantitative EEG results were found among subjects with delirium coexisting with dementia. The current study differs from the earlier work as we used the clinical examination diagnosis by DSM-III-R criteria as the standard of comparison, whereas Koponen used MMSE.

These findings also generally agree with those of Trzepacz et $a l,{ }^{12}$ who studied a less heterogeneous, younger sample of patients with hepatic encephalopathy. Both studies suggest that it is possible to develop discriminant functions based on selected EEG variables which reliably predict whether a subject is delirious. The variables identified by discriminant analysis might not only be useful in a cross-sectional differential diagnosis, but could also facilitate monitoring of response to treatment.

As this was a retrospective study involving a relatively small number of subjects, these results must be viewed with appropriate cau- tion, particularly as groups were not matched on important variables such as age, and a full spectrum of illness was not represented among control subjects, as shown by their uniformly high MMSE scores. In addition, inpatient versus outpatient status of the delirium and dementia groups is a potential confounder. Relevant to the latter point, Koponen et al found that the variables delta percentage and mean frequency were correlated with length of delirium and length of hospital stay, but those researchers did not comment specifically on hospital stay. ${ }^{13}$ Leuchter recently observed that EEG measures obtained from outpatients with dementia do not differ from those of inpatients with dementia (unpublished work). In fact, patient status in the current study simulates that in clinical practice; patients with delirium are admitted to hospital whilst those with dementia in the absence of acute illness are not.

Another potential confounder was medication status of the delirious versus non-delirious subjects, as medications are known to affect the EEG. In this limited patient sample, it turned out that medicated subjects in the delirium and combined disease groups were not outliers on the EEG and QEEG variables discussed. All variables for non-medicated subjects in the delirium and combined-disease groups were within two standard deviations of the means for medicated subjects. Koponen et al also noted in their study that there was no significant difference in spectral analysis results between delirious subjects treated with neuroleptics and delirious subjects who were unmedicated. It will be critical for future studies to account for medication status, since institutions certainly differ in types and doses of medications used to treat delirium.

The development of actual discriminant functions to distinguish encephalopathic from normal or "functional" states and delirium from dementia among the elderly must await replication of these results on a larger sample of subjects, preferably in a prospective study design in which groups could be better matched, and the effects of hospital admission and medication treatment examined concurrently. In such a study, it might be possible by EEG and QEEG findings to address more fundamental questions regarding the anatomical substrate of delirium, particularly if subjects in the combined disease group were matched to the delirium-only group as well as a dementia-only group. On the basis of the current pilot study, it is clear that EEG with quantitative analysis has the potential to provide important information to supplement the clinical examination in making an appropriate and timely diagnoses.

We acknowledge the contribution of Dr Herbert Weiner for critical review of this manuscript, as well as the technical critical review of this manuscript, as well as the technical assistance of Suzanne Hodgkin, Toni Saunders and Mariahn Smith, in performing these studies. This work was supported in A 40705 . SAJ was a Research Fellow supported by USPHS Award MH 17140 .

1 Lipowski ZJ. Delirium in the elderly patient. $N$ Engl $\mathcal{F}$ Med 1989;320:578-82.

2 Francis J, Martin D, Kapoor WN. A prospective study of 
delirium in hospitalized elderly. $\mathscr{J} A M A$ 1990;263: 1097-101.

3 Thomas RI, Cameron DJ, Fahs MC. A prospective study of delirium and prolonged hospital stay. Arch Gen Psychiatry 1988;45:937-40.

4 Lipowski ZI. Delirium: acute confusional states. New York: Oxford University Press, 1990:175-88.

5 American Psychiatric Association. Diagnostic and statistical manual of mental disorders, 3rd ed rev. Washington, DC: manual of mental disorders, 3rd ed

6 Folstein MF, Folstein SE, McHugh PR. "Mini-mental state": a practical method for grading the cognitive state state": a practical method for grading the cognitive state
of patients for the clinician. $\mathscr{J}$ Psychiatry Res 1975;12: of patients

7 Macdonald AJD, Simpson A, Jenkins D. Delirium in the elderly: a review and a suggestion for a research programme. Int $\mathcal{F}$ Geriat Psychiat 1989;4:311-19.

8 Romano J, Engel GL. Studies of delirium I: electroencephalographic data. Arch Neurol Psychiat 1944;51: 356-77.

9 Laidlaw J, Read AE. The electroencephalographic diagnosis of manifest and latent "delirium" with particular reference to that complicating hepatic cirrhosis. $f$ Neurol
Neurosurg Psychiatry 1961;24:58-70.

10 Obrecht R, Okhomina FOA, Scott DF. Value of EEG in acute confusional states. $\mathcal{F}$ Neurol Neurosurg Psychiatry 1979;52:980-5.

11 Jerrett SA, Corsak J. Clinical utility of topographic EEG brain mapping. Clin Electroenceph 1988;19(3):134-43.

12 Trzepacz PT, Brenner RP, Coffman G, van Thiel DH Delirium in liver transplantation candidates: discriminant analysis of multiple test variables. Biol Psychiatry 1988; 24:3-14.

13 Koponen H, Partanen J, Paakkonen A, Mattila E, Riekkinen PF. EEG spectral analysis in delirium. $\mathcal{f}$ Neurol Neurosurg 89:52:980-5.

14 Leuchter AF, Spar JE, Walter DO, Weiner H. Electroencephalographic spectra and coherence in the diagnosis of Alzheimer's-type and multi-infarct dementia. Arch Gen Psychiatry 1987;44:993-8.

15 Klass DD, Sharbrough FW. Electroencephalography. In Aronson E, Auger RG, Bastron JS, et al, eds. Clinical examinations in neurology. Mayo Clinic and Mayo Foundation 5th ed. Philadelphia: WB Saunders, 1981:278-99.

16 Dixon W. BMDP Biomedical Computer Programs. Berkeley, California: University of California Press, 1990. 